\title{
Downregulation of UBAP2L Inhibits the Epithelial-Mesenchymal Transition via SNAIL1 Regulation in Hepatocellular Carcinoma Cells
}

\author{
Tao Ye $e^{a}$ Jing $\mathrm{Xu}^{\mathrm{a}}$ Ling $\mathrm{Du}^{\mathrm{a}}$ Wenhui Mo $\mathrm{M}^{\mathrm{a}}$ Yiming Liang ${ }^{\mathrm{b}}$ Jinglin Xia ${ }^{\mathrm{a}, \mathrm{c}}$ \\ aMinhang hospital, Fudan University, Shanghai ' ${ }^{2}$ Shanghai Cancer Center, Fudan University, Shanghai, \\ ' Liver Cancer Institute, Zhongshan Hospital, and Key Laboratory of Carcinogenesis and Cancer Invasion \\ (Ministry of Education), Fudan University, Shanghai, China
}

\section{Key Words}

Hepatocellular carcinoma - Ubiquitin associated protein 2-like - Epithelial-mesenchymal transition $\cdot$ SNAIL1 $\cdot$ E-cadherin

\begin{abstract}
Background/Aims: Dysregulation of ubiquitin-associated protein 2-like (UBAP2L) has been reported in tumors, but its role in hepatocellular carcinoma $(\mathrm{HCC})$ progression is unclear. Methods: The expression levels of UBAP2L in HCC tissues and HCC cell lines were detected by western blot and quantitative real-time (qRT) PCR. The effects of UBAP2L expression on HCC cell biological traits, including migration and invasion, were investigated by wound healing assay and matrigel transwell assay. Simultaneously, the expression of epithelial-mesenchymal transition (EMT) markers including E-cadherin, $\mathrm{CK}-18, \mathrm{~N}$-cadherin, Vimentin, Claudin7 and the promoter activity of E-cadherin were detected by western blot and GRT-PCR. Subsequently, role of SNAIL1 in UBAP2L-mediated EMT and the mechanism underlying UBAP2L-mediated SNAIL1 expression were further investigated. Results: UBAP2L was overexpressed in human HCC tissues compared with peri-tumoral tissues. Downregulation of UBAP2L inhibited migration, invasion and the EMT in highly metastatic HCC cell lines. Furthermore, UBAP2L knockdown inhibited expression of the transcriptional repressor SNAIL1 and its ability to bind to the E-cadherin promoter via SMAD2 signaling pathway, which in turn resulted in increased E-cadherin expression. Additionally, bioinformatics analysis showed that expression of UBAP2L is correlated with poor prognosis in patients with HCC. Conclusions: UBAP2L plays a critical role in maintenance of the metastatic ability of HCC cells via SNAIL1 Regulation and is predictive of a poor clinical outcome.

\section{Introduction}

Hepatocellular carcinoma (HCC) is one of the most prevalent cancers and the third leading cause of cancer-related death [1,2]. Despite rapid developments in therapeutic management, including early screening, targeted drugs, local therapeutics and hepatectomy, the 5-year

T. Ye and J. Xu contribute to the article equally.

Jinglin Xia

Minhang hospital, Fudan university, Shanghai 170 Xin Song Road, Shanghai, 201199 (China)

E-Mail xiajinglin@fudan.edu.cn
\end{abstract}

KARGER 
survival rate has not improved significantly in nearly half a century. The aggressiveness of HCC is responsible for its rapid progression and low sensitivity to treatment. The epithelialmesenchymal transition (EMT) plays a pivotal role in local invasion and distant metastasis during HCC progression [3-7]. E-cadherin is a marker of epithelial cells and is critical for maintenance of a polar epithelium [8]. EMT initiation in HCC cells is marked by the loss of E-cadherin expression [9]. Previous studies have reported that a series of transcriptional factors-including SNAIL1, SNAIL2, ZEB1, ZEB2, TWIST and PRRX1-are involved in regulating E-cadherin expression and triggering the EMT [10-16]. However, the key events that act upstream of these factors in HCC are unclear.

Ubiquitin associated protein 2-like (UBAP2L) is a widely conserved protein with an ubiquitin-associated domain and multiple RGG/RG repeats at the N-terminus. UBAP2L interacts with the polycomb complex protein B lymphoma Moloney murine leukemia virus insertion region 1 (BMI1) to maintain hematopoietic stem cell activity [17] and plays an important role in the progression of mitosis by correcting kinetochore-microtubule attachment [18]. BMI1 is a well-known polycomb group (PcG) protein that has been reported to be associated with the EMT of cancer cells [19]. However, as a component of BMI1-containing protein complexes, whether UBAP2L plays a role in EMT is unclear. Recent studies have demonstrated that UBAP2L expression is increased in myeloma and HCC [20-22] and involved in the growth and migration of glioma and prostate cancer [23, 24]; however, the function of UBAP2L in HCC has not been investigated.

In the present study, we found that UBAP2L was highly expressed in the majority of HCC tissues and high-metastatic-potential HCC cell lines. Downregulation of UBAP2L significantly suppressed the migration, invasion and EMT of HCC cells in vitro by inhibiting the expression and binding of SNAIL1 to the E-cadherin promoter.

\section{Materials and Methods}

Tissue specimens

RNA and protein samples from human HCC and matching peri-tumoral normal tissues were collected from twelve patients who were admitted to Fudan University Shanghai Cancer Center. All patients underwent surgery with no chemotherapy, radiotherapy or any other adjuvant therapy. All samples were subsequently diagnosed as primary HCC by the Department of Pathology.

Ethics statement

Written informed consent was obtained from all subjects, and all protocols were approved by the Institutional Review Board of Fudan University.

Cell lines and cell culture

The HCCLM3, MHCC97H and MHCC97L cell lines were established at the Liver Cancer Institute of Fudan University and authenticated by short-tandem-repeat validation analysis during the study period. HepG2, SMMC-7721, Huh7, Hep3B and normal liver HL-7702 cells were purchased from the Cell Bank of China Academy of Sciences (Shanghai, China) and routinely checked for Mycoplasma contamination by Hoechst staining. Cell lines were cultured in high-glucose Dulbecco's modified Eagle's medium (DMEM; Gibco, USA) supplemented with 10\% fetal bovine serum (FBS) (Gibco, USA), $10 \mu \mathrm{g} / \mathrm{ml}$ streptomycin sulfate and $100 \mu \mathrm{g} / \mathrm{ml}$ penicillin G. Cells were incubated at 37 $\mathrm{C}$ in a humidified atmosphere containing $5 \% \mathrm{CO}_{2}$.

\section{RNA isolation and real-time PCR analysis}

Total RNA was extracted from fresh tissues and cells using TRIzol reagent (Invitrogen, CA, USA) according to the manufacturer's protocol. Total RNA (500 ng) was reverse-transcribed into complementary DNA using the Reverse Transcription Reagent Kit (TaKaRa, Japan). Real-time PCR analysis was performed using the 7500 Real-Time PCR system (Applied Biosystems, USA) with a SYBR Green PCR Amplification Kit (TaKaRa). Primers are shown in Table 1. Each PCR analysis was performed in triplicate, and the results were normalized to $\beta$ actin expression. The $2^{-\Delta \Delta \mathrm{Ct}}$ method was used for data analysis. 


\begin{tabular}{|c|c|}
\hline Cellular Physiology & Cell Physiol Biochem 2017;41:1584-1595 \\
\hline and Biochemistry & \begin{tabular}{l|l}
$\begin{array}{l}\text { DOI: 10.1159/000470824 } \\
\text { Publisned online: Marcn 27, } 2017 \text { The Author(s). Published by S. Karger AG, Basel } \\
\text { www.karger.com/cpb }\end{array}$ \\
\end{tabular} \\
\hline
\end{tabular}

Table 1. Primers for PCR used in this study

\begin{tabular}{|c|c|c|}
\hline Gene Name & Forward primer $\left(5^{\prime} \rightarrow 3^{\prime}\right)$ & Reverse primer $\left(5^{\prime} \rightarrow 3^{\prime}\right)$ \\
\hline UBAP2L & ATTCGCCTCACTCTCCACAC & TACCACCACACAACACAGCA \\
\hline E-cadherin & TGATTCTGCTGCTCTTGCTG & СТCTTCTCCGCСTCСTTCTT \\
\hline CK-18 & TCGCAAATACTGTGGACAATGC & GCAGTCGTGTGATATTGGTGT \\
\hline $\mathrm{N}$-cadherin & CGTGAAGGTTTGCCAGTGT & CAGCACAAGGATAAGCAGGA \\
\hline Vimentin & AGAGAACTTTGCCGTTGAAGC & ACGAAGGTGACGAGCCATT \\
\hline Claudin 7 & AGCTGCAAAATGTACGACTCG & GGAGACCACCATTAGGGCTC \\
\hline SNAIL1 & TTTACCTTCCAGCAGCCCTA & GACAGAGTCCCAGATGAGCA \\
\hline SNAIL2 & ACAGCGAACTGGACACACAT & GAATGGAGCAGCGGTAGTC \\
\hline ZEB1 & TGCCCAAACTGCAAGAAACG & TGAGTCCTGTTCTTGGTCGC \\
\hline ZEB2 & TTTCCTGCCCTCTCTGTAGC & CTCCTTGGGTTAGCATTTGG \\
\hline TWIST & AGCAAGATTCAGACCCTCAAG & ATCCTCCAGACCGAGAAGG \\
\hline BMI1 & CGTGTATTGTTCGTTACCTGGA & TTCAGTAGTGGTCTGGTCTTGI \\
\hline $\begin{array}{l}\text { E-cadherin } \\
\text { promoter }\end{array}$ & CCCACCACGTACAAGGGTC & ATGCCATCGTTGTTCACTGGA \\
\hline$\beta$-actin & CGTGGACATCCGTAAAGACC & ACATCTGCTGGAAGGTGGAC \\
\hline
\end{tabular}

Table 2. The sequences of shRNA used in this study

\begin{tabular}{|c|c|}
\hline shRNA & shRNA Sequence (5'-3') \\
\hline \multirow[t]{2}{*}{ Mock shRNA } & Sense: CCGGTTCTCCGAACGTGTCACGTTTCAAGAGAACGTGACACGTTCGGAGAATTTTTG \\
\hline & Antisense:AATTCAAAAATTCTCCGAACGTGTCACGTTCTCTTGAAACGTGACACGTTCGGAGAA \\
\hline \multirow[t]{2}{*}{ UBAP2L-shRNA1 } & Sense: CACCGCCAGCCAATACTGATGATAACGAATTATCATCAGTATTGGCTGGC \\
\hline & Antisense: AAAAGCCAGCCAATACTGATGATAATTCGTTATCATCAGTATTGGCTGGC \\
\hline \multirow[t]{2}{*}{ UBAP2L-sRNA2 } & Sense: CACCGCATGTTAGGGAAAGGATTTGCGAACAAATCCTTTCCCTAACATGC \\
\hline & Antisense: AAAAGCATGTTAGGGAAAGGATTTGTTCGCAAATCCTTTCCCTAACATGC \\
\hline \multirow[t]{2}{*}{ UBAP2L-shRNA3 } & Sense: CACCGGGAAGACACCATCTACAATGCGAACATTGTAGATGGTGTCTTCCC \\
\hline & Antisense: AAAAGGGAAGACACCATCTACAATGTTCGCATTGTAGATGGTGTCTTCCC \\
\hline
\end{tabular}

Western blot analysis

Total proteins from fresh tissues and cells were extracted using RIPA Protein Lysis solution (Pierce, IL, USA) and quantified by the Bradford method. Prepared samples were electrophoresed by sodium dodecyl sulfate polyacrylamide gel electrophoresis and blotted onto a polyvinylidene fluoride membrane (Millipore, MA, USA) using the Tetra Handcast system (Bio-Rad, USA). The membranes were blocked for $1 \mathrm{~h}$ at room temperature in Tris-buffered saline with $0.05 \%$ Tween (TBST) containing 5\% non-fat milk and incubated overnight at $4^{\circ} \mathrm{C}$ with the appropriate primary antibody. After washing in TBST, the membranes were incubated with the secondary antibody and visualized using an electrochemiluminescence kit (Pierce, IL, USA). The protein bands were detected and quantified using the Gene Gnome Syngene Bio Imaging System (Syngene, UK).

The primary anti-UBAP2L antibody was purchased from Abcam (MA, USA), and the anti- $\beta$ actin antibody was purchased from Santa Cruz Biotechnology Inc. (CA, USA). Other primary antibodies (anti-Ecadherin, anti-CK-18, anti-Claudin7, anti-N-cadherin, anti-vimentin, anti-SNAIL1, anti-STAT3, anti-p-STAT3, anti-P38 MAPK, anti-p-P38 MAPK, anti-SMAD2, anti-p-SMAD2, anti-P65, anti-p-P65, anti-JNK, and anti-pJNK) were purchased from Cell Signaling Pathway (MA, USA). Anti-mouse or anti-rabbit HRP-conjugated secondary antibodies were used (Santa Cruz Biotechnology Inc.).

\section{Lentivirus construction}

Lentivirus construction of UBAP2L-shRNA and mock-shRNA were purchased from Shanghai Genechem Company. The designed shRNA sequences are described in Table 2. Stable UBAP2L knockdown HCC cell lines were confirmed by quantitative real-time PCR and Western blot analysis.

Cell transfection

UBAP2L overexpression and SNAIL1 overexpression were performed by transfected with UBAP2L plasmid and SNAIL1 plasmid (GenePharma, China) using Lipofectamine 2000 (Invitrogen, USA) according KARGER 


\section{Cellular Physiology Cell Physiol Biochem 2017;41:1584-1595

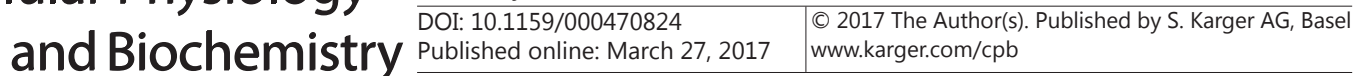 \\ Ye et al.: Role of UBAP2L in EMT}

to the manufacturer's instructions. Triplicate samples were run in all experiments, which were repeated at least three times.

\section{Wound-healing migration assays}

HCC cells were cultured until monolayer formation and then serum-starved for $12 \mathrm{~h}$. The cell monolayer was wounded using a $10 \mu \mathrm{l}$ plastic pipette tip. The remaining cells were washed twice with culture medium to remove cell debris and incubated at $37^{\circ} \mathrm{C}$ in normal serum-containing culture medium with the presence of mitomycin C $(10 \mathrm{ug} / \mathrm{ml})$ to avoid the influence of cell proliferation. At 0 and $48 \mathrm{~h}$, migrating cells at the wound front were photographed. The percentage of the cleared area at each time point compared with that at time zero was determined using the Image-Pro Plus v. 6.2 software.

\section{Cell migration and invasion assays}

Cell migration and invasion assays were performed using 24-well Transwell chambers (8 $\mu \mathrm{m}$ pore size; Millipore) precoated with or without $80 \mu \mathrm{l}$ Matrigel (1:5 dilution; BD Biosciences, San Jose, CA, USA). Briefly, $1 \times 10^{5}$ cells were suspended in $100 \mu \mathrm{l}$ serum-free medium with the presence of mitomycin C (10ug/ $\mathrm{ml}$ ), and added to the upper chamber, and $600 \mu \mathrm{l}$ DMEM supplemented with $20 \%$ FBS and $10 \mathrm{ug} / \mathrm{ml}$ of mitomycin $\mathrm{C}$ were placed in the lower chamber. After 24 or $48 \mathrm{~h}$ of incubation cells on the surface of the membrane were fixed in $4 \%$ paraformaldehyde and stained with $1 \%$ crystal violet. The Matrigel and cells remaining in the upper chamber were removed using cotton swabs. Cells in five microscopic fields were counted and photographed (200× magnification). All experiments were performed in triplicate.

\section{Luciferase reporter assays}

Luciferase reporter gene constructs containing intact E-cadherin promoter sequences or a E-cadherin promoter sequence with specific mutations in the E-box elements were described in detail previously [25, 26]. UBAP2L-shRNA stable HCC cells were cultured in 24-well plates and transfected with $1 \mu \mathrm{g}$ reporter gene constructs [E-cadherin (-108)-Luc, E-box-mutated E-cadherin (-108)-AMut-Luc, E-box-mutated E-cadherin (-108)-CMut-Luc, or E-box-mutated E-cadherin (-108)-ABCMut-Luc] using Lipofectamine 2000 (Invitrogen) according to the manufacturer's instructions. After $24 \mathrm{~h}$ of transfection, luciferase activities were measured using the Dual-Glo ${ }^{\mathrm{TM}}$ Luciferase Assay System kit (Promega). Luciferase intensities were normalized to the activity of Renilla luciferase. Triplicate samples were run in all experiments, which were repeated at least three times.

Chromatin Immunoprecipitation Assay

As described previously [27], HCC cells were fixed with 1\% formaldehyde and processed with the ChIPIT Enzymatic kit (Active Motif, Carlsbad, CA) following the manufacturer's protocol. The resulting protein/ DNA complexes were then immunoprecipitated overnight at $4^{\circ} \mathrm{C}$ using anti-SNAIL1 antibody(Abcam, MA, USA), non-specific IgG antibodies (Santa Cruz Biotechnology Inc., CA, USA), respectively, with protein G magnetic beads. The cross-linked protein/DNA complexes were eluted from $\mathrm{G}$ beads at $94^{\circ} \mathrm{C}$ for 15 minutes and then treated with proteinase $\mathrm{K}$ at $37^{\circ} \mathrm{C}$ for 1 hour to reverse protein/DNA complex. Stop buffer was subsequently added to inhibit proteinase $\mathrm{K}$ function. The PCRs were conducted using the primers for E-cadherin promoter (Table 1).

\section{Survival analysis}

Univariate associations between expression profiles and survival were reassessed by Cox regression using the coxph function in the R statistical software package "survival" [28]. Differences between survival curves and log rank P values were reassessed using the survdiff function of the "survival" package. Clinical survival information and normalized RNA-Seq expression data of tumor samples were downloaded from Broad GDAC FIREHOSE on 2014-12-06 (http://gdac.broadinstitute.org/runs/stddata_2014_12_06/). Only 339 cases of HCC had both RNA-Seq expression data and corresponding clinical information available. These data were used to build survival models.

\section{Statistical analysis}

Statistical analysis was performed using SPSS16.0 statistical software (SPSS Inc., Chicago, IL, USA). Student's $t$-tests were used to assess significant differences among study groups. The level of significance was set at $\mathrm{p}<0.05$. All experiments were performed at least in triplicate.

\section{KARGER}




\begin{tabular}{|c|c|}
\hline Cellular Physiology & Cell Physiol Biochem 2017;41:1584-1595 \\
\hline and Biochemistry & 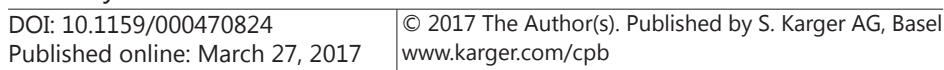 \\
\hline
\end{tabular}

\section{Results}

UBAP2L expression is frequently upregulated in human HCCs

The UBAP2L mRNA level in HCC tissues was evaluated by ONCOMINE data-mining analysis. UBAP2L expression was significantly higher in human HCCs compared with normal liver tissue (Fig. 1A). UBAP2L expression was next analyzed in human HCC tissues and matched peri-tumor tissues by western blot and quantitative real-time (qRT) PCR analyses. UBAP2L mRNA and protein levels were higher in 9/12 HCC tissues than in matched peritumor tissues (Fig. 1B and 1C). Additionally, we investigated UBAP2L expression in various HCC cell lines. LM3 and 97H cells have greater metastatic potential than that of other HCC cell lines (97L, Hep3B, Huh7 and HepG2) or normal liver L02 cells. UBAP2L expression was significantly higher in the highly metastatic HCC cell lines (Fig. 1D). These results suggest that UBAP2L expression is frequently upregulated in human HCCs.

Downregulation of UBAP2L inhibits the migration and invasion of HCC cells

To further explore the biological significance of UBAP2L in HCC, we performed shRNAmediated stable UBAP2L knockdown in LM3 and 97H cells, and overexpressed UBAP2L in L-02 cells. The efficacy of UBAP2L down- and up-regulation was confirmed by qRT-PCR and western blot analyses (Fig. 2A, 2B, and 2D). UBAP2L-knockdown cells exhibited an epitheliallike morphology, rather than the mesenchymal spindle-like morphology characteristic of LM3-mock and 97H-mock cells (Fig. 2C). While upregulation of UBAP2L in L-02 cells exhibited the opposite phenotype (Fig. 2E). The results indicated that UBAP2L is necessary for maintenance of the mesenchymal morphology and may affect the EMT. shRNA1 resulted in $80 \%$ downregulation of UBAP2L expression, while shRNA2 and shRNA3 resulted in 50\% of that. Therefore, shRNA1 was chosen to further studying. We investigated the migration and invasiveness of HCC cells and L-02 cells with the presence of mitomycin C (10ug/ml). In wound-healing migration assays, LM3-shRNA1 and 97H-shRNA1 cells exhibited a significant delay in migration at $48 \mathrm{~h}$ post-wounding compared with control cells (Fig. 3A). Similarly,

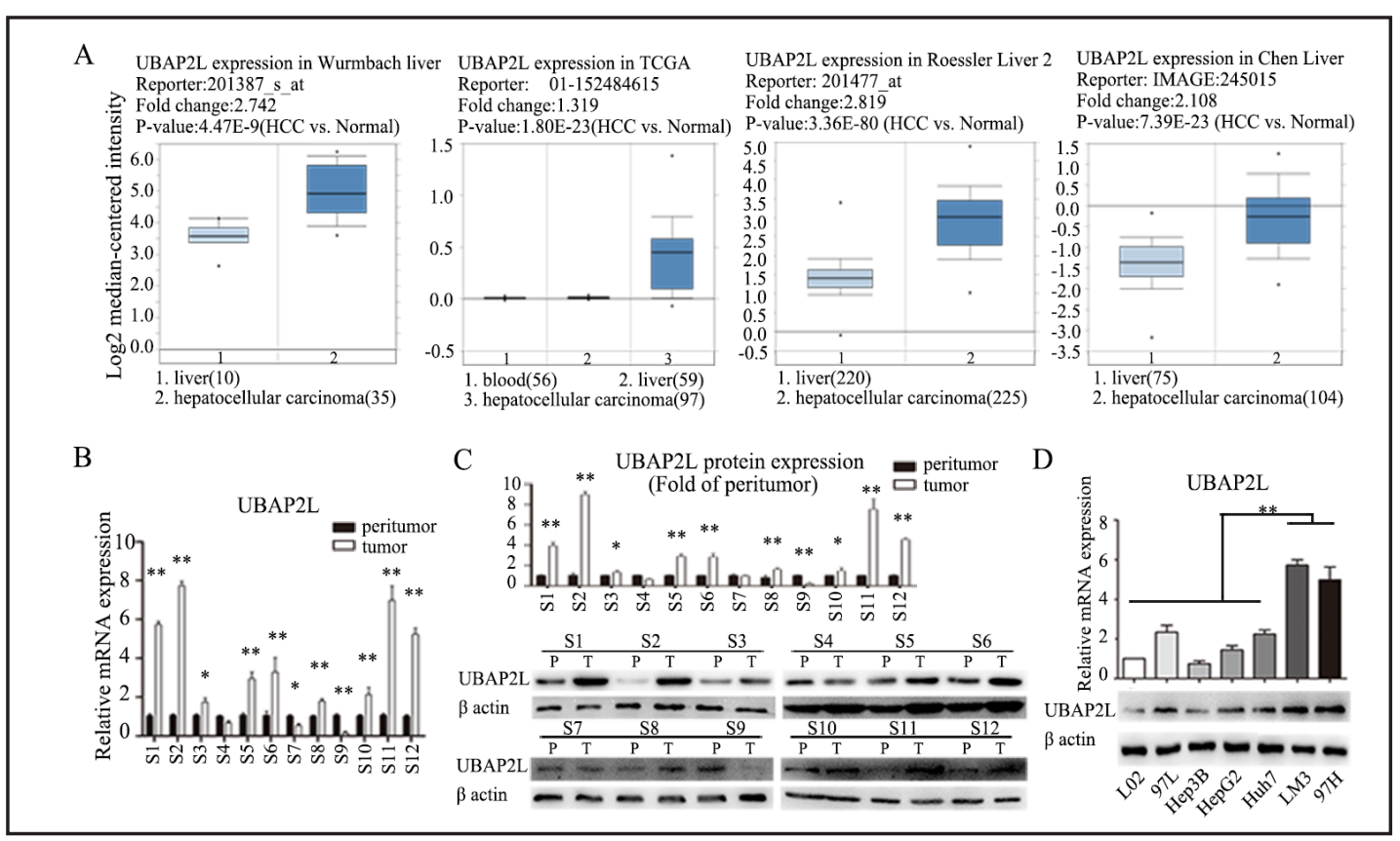

Fig. 1. UBAP2L expression is frequently upregulated in human HCCs. (A) Oncomine plots of UBAP2L mRNA levels in HCC tissues and normal liver tissues obtained from public HCC datasets (Wurmback, Roessler, Chen, and TCGA dataset). (B, C) UBAP2L expression in human HCC tissues and matching peri-tumoral tissues. (D) UBAP2L expression in HCC cells with different metastatic capabilities and normal liver L02 cells. Data represent the mean $\pm \mathrm{SD}$ of triplicate samples; ${ }^{*} \mathrm{P}<0.05$, ${ }^{* *} \mathrm{P}<0.01$. 
Fig. 2. Morphological analysis of UBAP2L-knockdown HCC cells and UBAP2L-upregulated L-02 cells. (A, B) Knockdown of UBAP2L by shRNA was confirmed by qRT-PCR and western blot. (C) Morphological analysis of UBAP2L-knockdown HCC cells. (D) Upregulation of UBAP2L in L-02 cells was confirmed by qRT-PCR and western blot. (E) Morphological analysis of UBAP2L-upregulated L-02 cells. Data represent the mean \pm $\mathrm{SD}$ of triplicate samples; ${ }^{* *} \mathrm{P}<0.01$. in transwell invasion assays, the numbers of invasive LM3-mock and 97H-mock cells were significantly higher than those of LM3-shRNA1 and 97H-shRNA1 cells (Fig. 3B). While upregulation of UBAP2L in L-02 cells exhibited the opposite phenotype (Fig. 3C and 3D). These results indicate that downregulation of UBAP2L inhibits HCC cell migration and invasion in vitro.

UBAP2L regulates EMT and E-cadherin expression in HCC cells

EMT is critical for local invasion and distant metastasis of cancer. To further confirm the role of UBAP2L in the EMT, the expression of EMT-related markers was detected. We found that UBAP2L-knockdown in LM3 and 97H cells upregulated the expression of epithelial markers (E-cadherin, CK-18) and downregulated that of mesenchymal markers (N-cadherin, vimentin, and Claudin7) (Fig. 4A-4C). Moreover, UBAP2L inhibition resulted in an at least twofold increase in E-cadherin promoter activity (Fig. 4D).

E-box elements in the E-cadherin promoter play a critical negative regulatory role in E-cadherin gene transcription. To explore the effect of UBAP2L knockdown on E-cadherin activity, we assayed promoter activity using luciferase reporter gene constructs with specific mutations in the E-box elements [E-box-mutated E-cadherin (-108)-AMut-Luc, E-boxmutated E-cadherin (-108)-CMut-Luc, or E-box-mutated E-cadherin (-108)-ABCMut-Luc]. As shown in Figure 4E, UBAP2L-knockdown failed to increase the activity of the E-cadherin promoter with a mutation in the E-box element (Fig. 4E). Therefore, UBAP2L inhibition enhances E-cadherin promoter activity in a manner dependent on intact proximal E-box elements.

UBAP2L knockdown inhibits the SNAIL1 expression via activating SMAD2 signaling pathway

Several key transcription factors-including SNAIL1, SNAIL2, ZEB1, ZEB2, TWIST and PRRX1-act as transcriptional inhibitors of E-cadherin expression and induce the EMT. We next investigated whether UBAP2L regulates the levels of these transcriptional factors. 


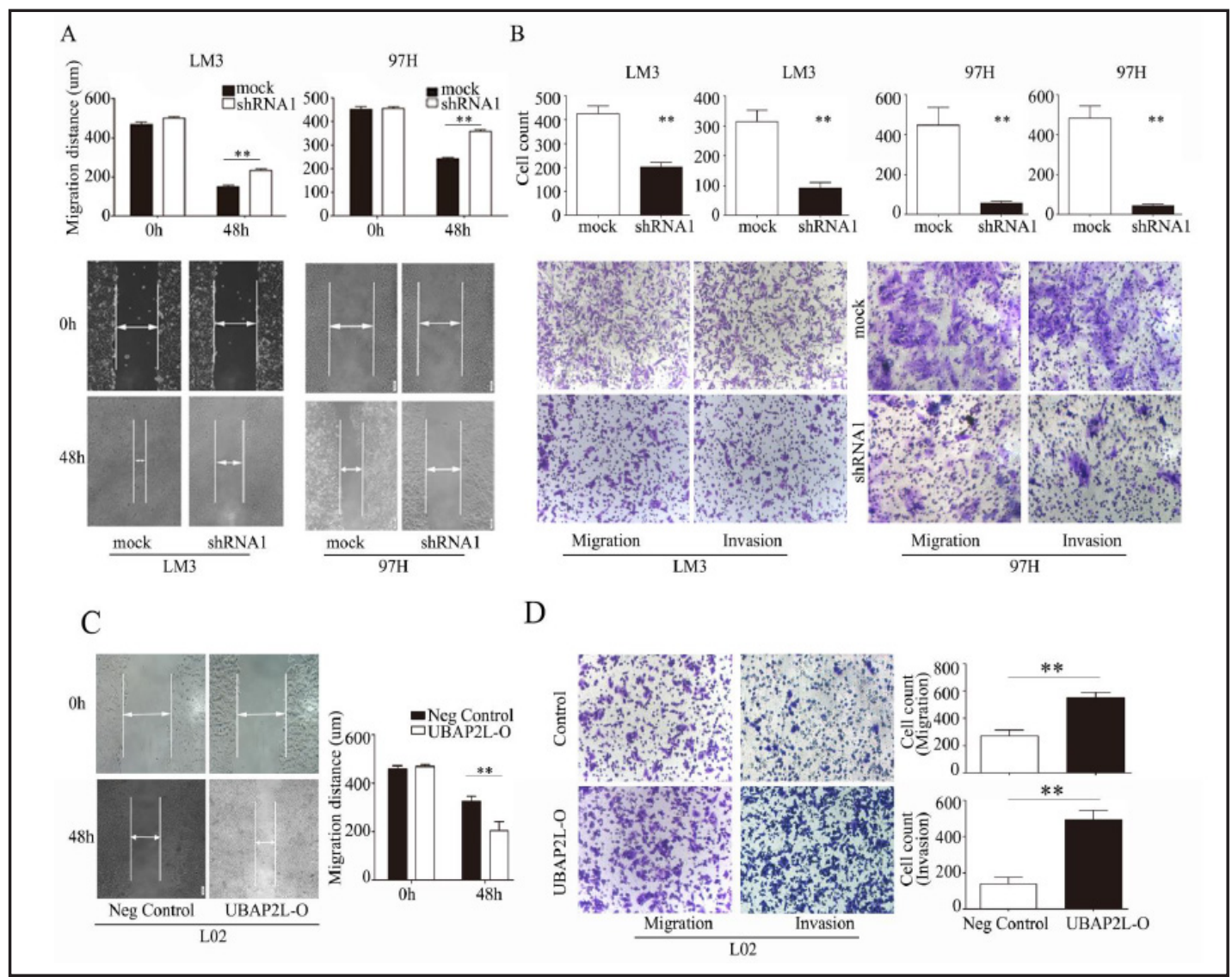

Fig. 3. Downregulation of UBAP2L inhibits the migration and invasion of HCC cells. (A) Wound-healing migration assays and quantification of distance of open area. Data are means \pm SD of triplicate samples; $* * \mathrm{P}<0.01$. Magnification, $\times 200$. (B) Migration and invasion after knockdown of UBAP2L in HCC cells were assessed using transwell assays with the presence of mitomycin C $(10 \mathrm{ug} / \mathrm{ml})$. Migration time is $48 \mathrm{~h}$ and invasion time is $72 \mathrm{~h}$. Data are means $\pm \mathrm{SD}$ of triplicate samples; ${ }^{* *} \mathrm{P}<0.01$. Magnification, $\times 100$. (C) Wound-healing migration assays and quantification of distance of open area in L-02 cells with UBAP2L overexpression. Data are means \pm SD of triplicate samples; ${ }^{* *} \mathrm{P}<0.01$. Magnification, $\times 200$. (D) Migration and invasion after overexpression of UBAP2L in L-02 cells were assessed using transwell assays with the presence of mitomy$\operatorname{cin~C~}(10 \mathrm{ug} / \mathrm{ml})$. Migration time is $48 \mathrm{~h}$ and invasion time is $72 \mathrm{~h}$. Data are means \pm SD of triplicate samples; $* * \mathrm{P}<0.01$. Magnification, $\times 100$.

Western blot and qRT-PCR analyses showed that SNAIL1 levels were significantly decreased after downregulation of UBAP2L in LM3 and 97H cells, and increased after upregulation in L-02 cells (Fig. 5A-G), suggesting SNAIL1 to be critical for UBAP2L-metiated E-cadherin expression. To validate this hypothesis, we investigated whether the inhibition of SNAIL1 by UBAP2L knockdown is associated with decreased binding to the E-cadherin promoter. As determined by chromatin immunoprecipitation assays (Fig. 5H-I), an anti-SNAIL1 antibody, but not a nonrelated immunoglobulin G, pulled down the E-box region of the E-cadherin promoter. Therefore, UBAP2L knockdown markedly decreased binding of SNAIL1 to the E-cadherin promoter. Additionally, we overexpressed SNAIL1 in stable UBAP2L-knockdown cells, and we found that SNAIL1 rescued the effect of UBAP2L knockdown on the EMT markers (Fig. 5J). The data suggested that Snail1 is critical for UBAP2L-mediated EMT.

BMI1 is a well-known polycomb group (PcG) protein that has been reported to be associated with the EMT of cancer cells [19]. We hypothesis that UBAP2L, as a component of BMI1-containing protein complexes, mediates SNAIL1 expression via regulating BMI1. However, BMI1 expression is not affected by UBAP2L-knockdown or overexpression (Fig. 
Fig. 4. Knockdown of UBAP2L in HCC cells inhibits EMT and promotes E-cadherin promoter activation. (A, B, C) qRT-PCR and western blot analysis of EMT marker expression. (D) The effect of UBAP2L knockdown on E-cadherin promoter activity in HCC cells. (E) E-cadherin promoter activity of E-box mutant constructs in HCC cells transfected with the empty vector and in stable UBAP2L-knockdown HCC cells. Data are means \pm SD of triplicate samples; ${ }^{* *} \mathrm{P}<0.01$.

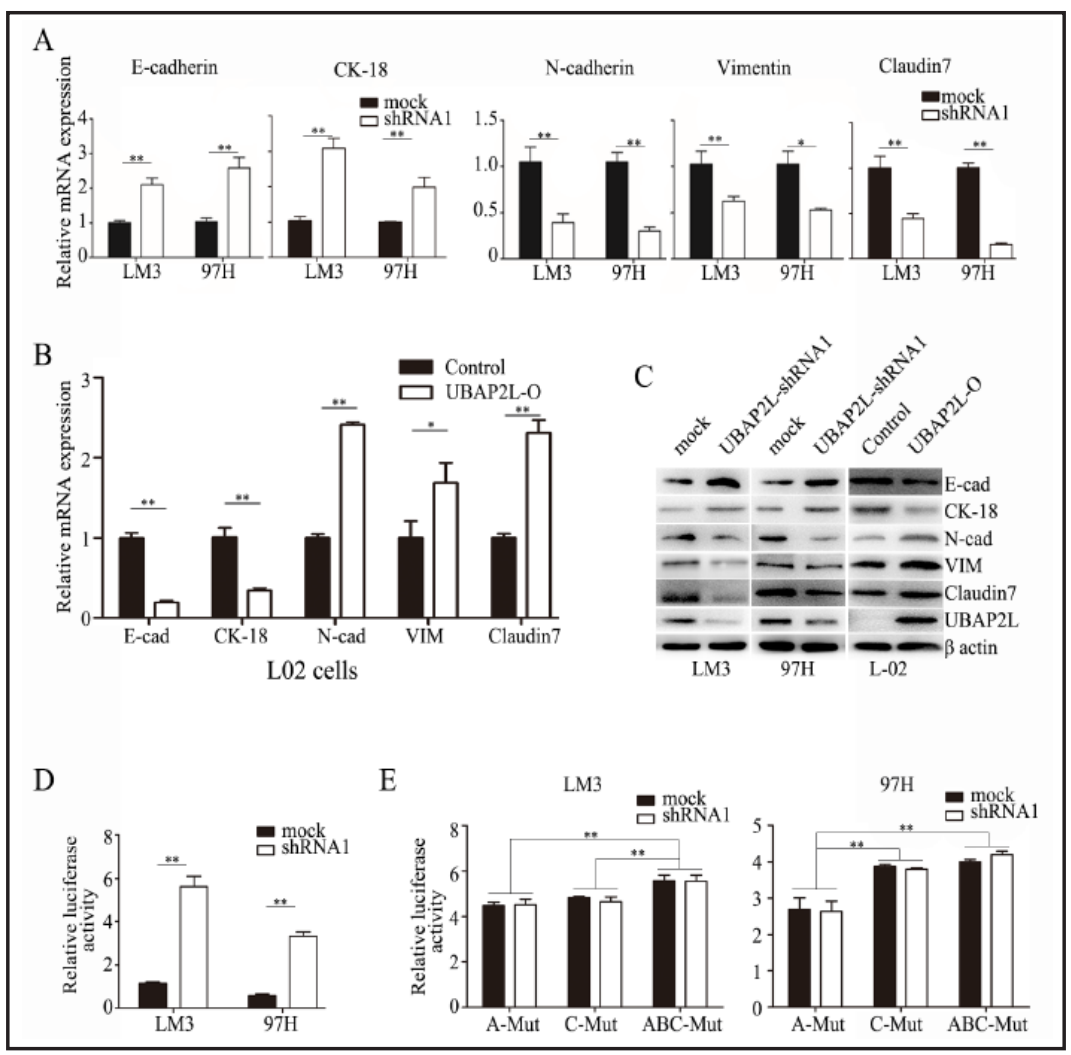

5G). To further explore the mechanism underlying UBAP2L-mediated Snail1 expression, we detected the phosphorylation state of STAT3, p38 MAPK, JNK, p65, SMAD2 signaling pathways which were involved in SNAIL1 expression and the EMT. We found that downregulation of UBAP2L inhibited the phosphorylation levels of SMAD2, and up-regulation of UBAP2L in L-02 cells activated the phosphorylation of SMAD2 (Fig. 5K). The data suggests that UBAP2L regulates the SNAIL1 expression via SMAD2 signaling pathway.

Expression of UBAP2L is correlated with a poor prognosis in patients with HCC

To assess the significance of UBAP2L expression in HCC prognosis, we downloaded HCC gene expression data and clinical information from the TCGA database (https://tcga-data. nci.nih.gov). Only 339 cases of HCC had both RNA-Seq expression data and corresponding clinical information available. These data were used to generate survival models. As shown in Figure 6, the overall survival rates of UBAP2 $L^{\text {high }}$ patients with HCC were significantly lower than those of UBAP2 $\mathrm{L}^{\text {low }}$ patients (Fig. 6), suggesting that UBAP2L expression might be a biomarker of a poor prognosis in patients with HCC.

\section{Discussion}

The molecular mechanism by which cancer cells undergo the EMT are unclear. In the present study, we demonstrated that UBAP2L was expressed at higher levels in HCC tissues compared with matched peri-tumoral tissues. Knockdown of UBAP2L significantly inhibited the EMT by downregulating SNAIL1 expression and binding to the E-cadherin promoter. Omics data analysis showed that UBAP2L expression is correlated with a poor prognosis in patients with HCC.

Local invasion and distant metastasis are responsible for the poor prognosis of patients with HCC. The 5-year survival rate of patients with HCC with regional invasion is $7 \%$, and that of those with distant metastasis is 3\% [29]. The EMT has been observed in various subsets of cancer cells and is essential for successful migration and seeding $[10,30]$. In 
Fig. 5. UBAP2L knockdown inhibits the expression and binding of SNAIL1 to the E-cadherin promoter. (A-G) Expression of the transcriptional repressors SNAIL1, SNAIL2, ZEB1, ZEB1 and TWIST was evaluated in HCC cells transfected with the empty vector and in UBAP2L-knockdown HCC cells. (H-I) Chromatin immunoprecipitation assay showing pull down of the E-box region of the E-cadherin promoter by anti-SNAIL1 antibodies. UBAP2L knockdown markedly decreased SNAIL1 binding to the E-cadherin promoter. (J) SNAIL1 was overexpressed in stable UBAP2L-knockdown cells, and rescued the effect of UBAP2L knockdown on EMT markers. (K) The phosphorylation state of STAT3, p38 MAPK, JNK, p65, SMAD2 signaling pathways were determined by western blot. Data represent the mean $\pm \mathrm{SD}$ of triplicate samples; ${ }^{*} \mathrm{P}<0.05$, $* * \mathrm{P}<0.01$.

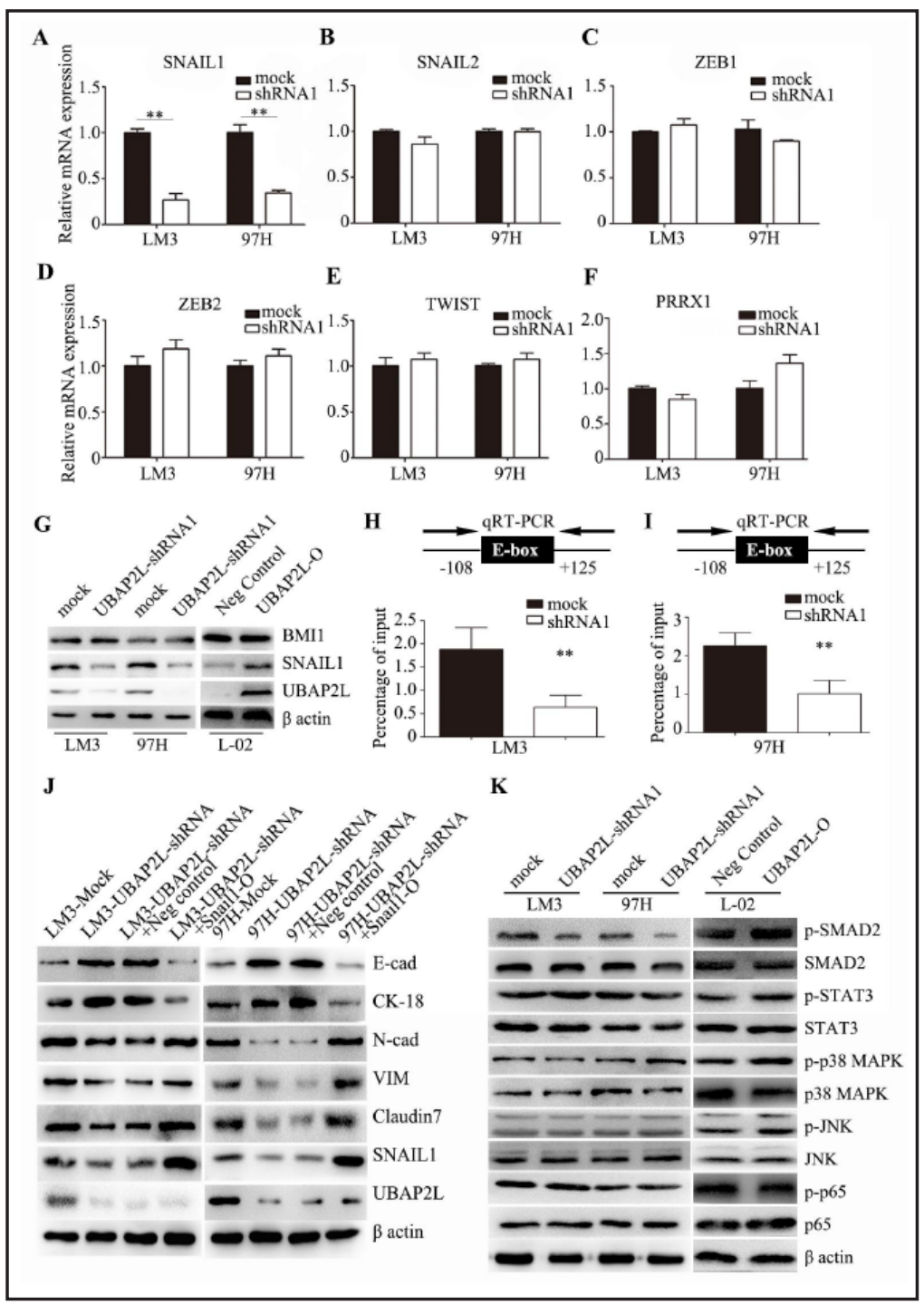

this study, UBAP2L knockdown resulted in an epithelial-like morphology, rather than the mesenchymal spindle-like morphology characteristic of HCC cells. Wound-healing migration and invasion assays also indicated that UBAP2L knockdown inhibited invasion by HCC cells.

The loss of epithelial cell junction proteins, such as E-cadherin, and gain of mesenchymal markers, such as vimentin and $\mathrm{N}$-cadherin, are critical for initiating the EMT [31]. We thus determined the effect of UBAP2L knockdown on these markers. The results indicated a marked increase in E-cadherin mRNA and protein levels and

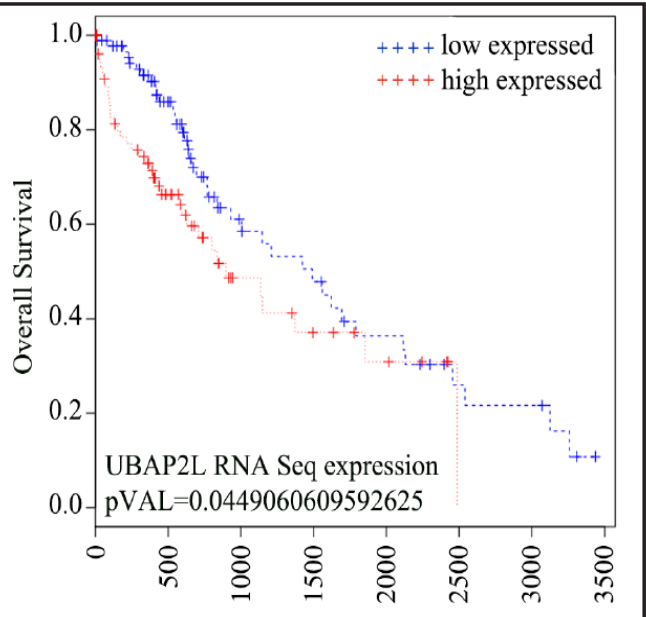

a decrease in $\mathrm{N}$-cadherin and vimentin Fig. 6. Overall survival rates of UBAP2 $\mathrm{L}^{\text {high }}$ patients expression. Moreover, E-cadherin promoter with HCC were significantly lower than those of activity was significantly increased by UBAP2L ${ }^{\text {low }}$ patients. 
UBAP2L knockdown. The E-cadherin promoter contains three E-boxes (A, B, C), a CCAAT box and a GC-rich element [26]. The E-box sequences mediate strong transcriptional repression of E-cadherin expression [32]. E-box $\mathrm{C}$ has been reported to play a more important role in repression of E-cadherin gene transcription than has E-box A, while E-box B does not modulate E-cadherin expression [26]. Thus, we generated three mutant reporter gene constructs: E-box A, E-box C and E-box ABC. Luciferase activity was higher in the E-box C mutant and E-box $\mathrm{ABC}$ mutant than in the E-box A mutant, in accordance with previous reports. Additionally, UBAP2L knockdown failed to modulate the activity of these mutant reporter genes, suggesting an important role for E-boxes in UBAP2L-mediated E-cadherin expression.

Transcriptional repression by factors such as SNAIL1, SNAIL2, ZEB1, ZEB2, TWIST and PRRX1 is a common mechanism of loss of E-cadherin expression [10-14]. SNAIL1, SNAIL2, ZEB1 and ZEB2 are zinc-finger transcription factors that bind E-box elements and repress endogenous E-cadherin expression [26, 33, 34]. TWIST, a highly conserved basic helixloop-helix transcription factor, plays a key role in repression of E-cadherin expression [35]. PRRX1, a EMT inducer, enhances the mesenchymal and stem-like phenotype of cancer cells [36]. Upregulation of these transcriptional repressors and downregulation of E-cadherin have been reported in several human cancers. In this study, we found that SNAIL1 mRNA and protein levels were significantly altered by UBAP2L. Moreover, the binding of SNAIL1 to the E-cadherin promoter was inhibited by UBAP2L knockdown. These results suggest that SNAIL1 is critical for UBAP2L-mediated EMT in HCC cells.

SMAD2 pathway is the canonical TGF $\beta$ signaling pathway, which is a crucial regulator of EMT and plays critical role in cancer progression [37]. Snail1 expression is also regulated by SMAD2 pathway in previous reports [38, 39]. We showed herein that UBAP2L enhanced the phosphorylation of SMAD2, suggesting a potential role of SMAD2 signaling pathway in UBAP2L-regulated Snail1 expression and EMT. The ligand TGF $\beta$ activates receptor serine kinases on the plasma membrane, which phosphorylate SMAD proteins and translocate it to the nucleus. Receptor-mediated signal-transduction pathway leads to multi-ubiquitination and degradation of SMAD2 by the proteasome. If the degradation is averted, phosphorylated SMAD2 would accumulate in the nucleus in active state $[40,41]$. UBAP2L is a protein involved in ubiquitin conjugation. We hypothesis that UBAP2L is involved in the process of SMAD2 ubiquitination. However, the concrete function of UBAP2L in ubiquitination is still obscure. Further investigation of the mechanism by which UBAP2L increased SMAD2 phosphorylation would be conducted in our subsequent work.

In conclusion, we have experimentally demonstrated that downregulation of UBAP2L inhibits the migration, invasion and EMT of HCC cells by regulating the binding of SNAIL1 to the E-cadherin promoter. UBAP2L expression is correlated with a poor prognosis of patients with HCC and could thus serve as a novel prognostic indicator.

\section{Funding}

This study was sponsored by grants from the National Natural Science Foundation of China (No. 81572395), the Shanghai Leading Academic Discipline Project (Project Number: B115), and the Shanghai Outstanding Academic Leaders (Project Number: 14XD1401100).

\section{Disclosure Statement}

The authors declare no commercial or financial conflict of interest. 


\section{Cellular Physiology Cell Physiol Biochem 2017;41:1584-1595

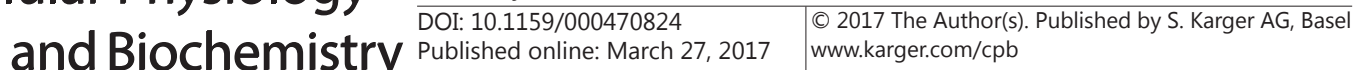

Ye et al.: Role of UBAP2L in EMT

\section{References}

1 Venook AP, Papandreou C, Furuse J, de Guevara LL: The incidence and epidemiology of hepatocellular carcinoma: a global and regional perspective. Oncologist 2010;15:5-13.

2 Singh S, Singh PP, Roberts LR, Sanchez W: Chemopreventive strategies in hepatocellular carcinoma. Nat Rev Gastroenterol Hepatol 2014;11:45-54.

3 Xiao S, Chang RM, Yang MY, Lei X, Liu X, Gao WB, Xiao JL, Yang LY: Actin-like 6A predicts poor prognosis of hepatocellular carcinoma and promotes metastasis and epithelial-mesenchymal transition. Hepatology 2016;63:1256-1271.

4 Fang JH, Zhou HC, Zhang C, Shang LR, Zhang L, Xu J, Zheng L, Yuan Y, Guo RP, Jia WH, Yun JP, Chen MS, Zhang Y, Zhuang SM: A novel vascular pattern promotes metastasis of hepatocellular carcinoma in an epithelialmesenchymal transition-independent manner. Hepatology 2015;62:452-465.

-5 Liu L, Dai Y, Chen J, Zeng T, Li Y, Chen L, Zhu YH, Li J, Li Y, Ma S, Xie D, Yuan YF, Guan XY: Maelstrom promotes hepatocellular carcinoma metastasis by inducing epithelial-mesenchymal transition by way of Akt/GSK-3beta/Snail signaling. Hepatology 2014;59:531-543.

6 Zhu H, Zhou X, Ma C, Chang H, Li H, Liu F, Lu J: Low Expression of miR-448 Induces EMT and Promotes Invasion by Regulating ROCK2 in Hepatocellular Carcinoma. Cell Physiol Biochem 2015;36:487-498.

7 Dong L, Ni J, Hu W, Yu C, Li H: Upregulation of Long Non-Coding RNA PlncRNA-1 Promotes Metastasis and Induces Epithelial-Mesenchymal Transition in Hepatocellular Carcinoma. Cell Physiol Biochem 2016;38:836-846.

8 Wheelock MJ: Catenin association with E-cadherin changes with the state of polarity of HT-29 cells. Exp Cell Res 1990;191:186-193.

-9 Yook JI, Li XY, Ota I, Fearon ER, Weiss SJ: Wnt-dependent regulation of the E-cadherin repressor snail. J Biol Chem 2005;280:11740-11748.

10 Ding W, You H, Dang H, LeBlanc F, Galicia V, Lu SC, Stiles B, Rountree CB: Epithelial-to-mesenchymal transition of murine liver tumor cells promotes invasion. Hepatology 2010;52:945-953.

11 Tang B, Qi G, Tang F, Yuan S, Wang Z, Liang X, Li B, Yu S, Liu J, Huang Q, Wei Y, Zhai R, Lei B, Yu H, Tomlinson S, He S: Aberrant JMJD3 expression upregulates Slug to promote migration, invasion and stem cell-like behaviors in hepatocellular carcinoma. Cancer Res DOI:10.1158/0008-5472.

12 Lv X, Li L, Lv L, Qu X, Jin S, Li K, Deng X, Cheng L, He H, Dong L: HOXD9 promotes epithelial-mesenchymal transition and cancer metastasis by ZEB1 regulation in hepatocellular carcinoma. J Exp Clin Cancer Res 2015;34:133.

13 Yang X, Wang J, Qu S, Zhang H, Ruan B, Gao Y, Ma B, Wang X, Wu N, Li X, Dou K, Li H: MicroRNA-200a suppresses metastatic potential of side population cells in human hepatocellular carcinoma by decreasing ZEB2. Oncotarget 2015;6:7918-7929.

14 Zhao N, Sun H, Sun B, Zhu D, Zhao X, Wang Y, Gu Q, Dong X, Liu F, Zhang Y, Li X: miR-27a-3p suppresses tumor metastasis and VM by down-regulating VE-cadherin expression and inhibiting EMT: an essential role for Twist-1 in HCC. Sci Rep 2016;6:23091.

15 Sanchez-Tillo E, Liu Y, de Barrios O, Siles L, Fanlo L, Cuatrecasas M, Darling DS, Dean DC, Castells A, Postigo A: EMT-activating transcription factors in cancer: beyond EMT and tumor invasiveness. Cell Mol Life Sci 2012;69:3429-3456.

-16 Wu W, Ding H, Cao J, Zhang W: FBXL5 inhibits metastasis of gastric cancer through suppressing Snail1. Cell Physiol Biochem 2015;35:1764-1772.

17 Bordeleau ME, Aucagne R, Chagraoui J, Girard S, Mayotte N, Bonneil E, Thibault P, Pabst C, Bergeron A, Barabe F, Hebert J, Sauvageau M, Boutonnet C, Meloche S, Sauvageau G: UBAP2L is a novel BMI1-interacting protein essential for hematopoietic stem cell activity. Blood 2014;124:2362-2369.

18 Maeda M, Hasegawa H, Sugiyama M, Hyodo T, Ito S, Chen D, Asano E, Masuda A, Hasegawa Y, Hamaguchi $\mathrm{M}$, Senga T: Arginine methylation of ubiquitin-associated protein 2-like is required for the accurate distribution of chromosomes. FASEB J 2016;30:312-323.

19 Ren H, Du P, Ge Z, Jin Y, Ding D, Liu X, Zou Q: TWIST1 and BMI1 in Cancer Metastasis and Chemoresistance. J Cancer 2016;7:1074-1080.

20 Naz RK, Dhandapani L: Identification of human sperm proteins that interact with human zona pellucida3 (ZP3) using yeast two-hybrid system. J Reprod Immunol 2010;84:24-31. 


\section{Cellular Physiology Cell Physiol Biochem 2017;41:1584-1595

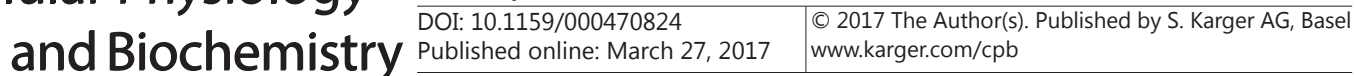

Ye et al.: Role of UBAP2L in EMT

21 Sawyer JR: The prognostic significance of cytogenetics and molecular profiling in multiple myeloma. Cancer Genet 2011;204:3-12.

22 Zhu ZZ, Wang D, Cong WM, Jiang H, Yu Y, Wen BJ, Dong H, Zhang X, Liu SF, Wang AZ, Zhu G, Hou L: Sexrelated differences in DNA copy number alterations in hepatitis B virus-associated hepatocellular carcinoma. Asian Pac J Cancer Prev 2012;13:225-229.

23 Zhao B, Zong G, Xie Y, Li J, Wang H, Bian E: Downregulation of ubiquitin-associated protein 2-like with a short hairpin RNA inhibits human glioma cell growth in vitro. Int J Mol Med 2015;36:1012-1018.

24 Li D, Huang Y: Knockdown of ubiquitin associated protein 2-like inhibits the growth and migration of prostate cancer cells. Oncol Rep 2014;32:1578-1584.

-25 Huang W, Zhang Y, Varambally S, Chinnaiyan AM, Banerjee M, Merajver SD, Kleer CG: Inhibition of CCN6 (Wnt-1-Induced Signaling Protein 3) Down-Regulates E-Cadherin in the Breast Epithelium through Induction of Snail and ZEB1. Am J Pathol 2008;172:893-904.

-26 Hajra KM, Chen DY, Fearon ER: The SLUG zinc-finger protein represses E-cadherin in breast cancer. Cancer Res 2002;62:1613-1618.

-27 Yao X, Ireland SK, Pham T, Temple B, Chen R, Raj MH, Biliran H: TLE1 promotes EMT in A549 lung cancer cells through suppression of E-cadherin. Biochem Biophys Res Commun 2014;455:277-284.

28 Therneau T: A Package for Survival Analysis in S. R package version 2.37-7. 2014;http://CRAN.R-project. org/package=survival.

29 Howlader N, Noone AM, Krapcho M, Garshell J, Miller D, Altekruse SF, Kosary CL, Yu M, Ruhl J, Tatalovich Z, Mariotto A, Lewis DR, Chen HS, Feuer EJ, Cronin KA (eds). SEER Cancer Statistics Review, 1975-2011, National Cancer Institute. Bethesda, MD, http://seer.cancer.gov/csr/1975_2011/.

30 Nieto MA, Huang RY, Jackson RA, Thiery JP: EMT: 2016. Cell 2016;166:21-45.

-31 Thiery JP, Acloque H, Huang RY, Nieto MA: Epithelial-mesenchymal transitions in development and disease. Cell 2009;139:871-890.

32 Giroldi LA, Bringuier PP, de Weijert M, Jansen C, van Bokhoven A, Schalken JA: Role of E boxes in the repression of E-cadherin expression. Biochem Biophys Res Commun 1997;241:453-458.

33 Comijn J, Berx G, Vermassen P, Verschueren K, van Grunsven L, Bruyneel E, Mareel M, Huylebroeck D, van Roy F: The two-handed E box binding zinc finger protein SIP1 downregulates E-cadherin and induces invasion. Mol Cell 2001;7:1267-1278.

-34 Grooteclaes ML, Frisch SM: Evidence for a function of CtBP in epithelial gene regulation and anoikis. Oncogene 2000;19:3823-3828.

- 35 Gao Y, Xuan XY, Zhang HY, Wang F, Zeng QR, Wang ZQ, Li SS: Relationship between TWIST expression and epithelial-mesenchymal transition of oesophageal squamous cell carcinoma. Cell Biol Int 2012;36:571577.

-36 Takahashi Y, Sawada G, Kurashige J, Uchi R, Matsumura T, Ueo H, Takano Y, Akiyoshi S, Eguchi H, Sudo T, Sugimachi K, Doki Y, Mori M, Mimori K: Paired related homoeobox 1, a new EMT inducer, is involved in metastasis and poor prognosis in colorectal cancer. Br J Cancer 2013;109:307-311.

- 37 Kim J, Kong J, Chang H, Kim H, Kim A: EGF induces epithelial-mesenchymal transition through phosphoSmad2/3-Snail signaling pathway in breast cancer cells. Oncotarget 2016;7:85021-85032.

-38 Wu Y, Fu Y, Zheng L, Lin G, Ma J, Lou J, Zhu H, He Q, Yang B: Nutlin-3 inhibits epithelial-mesenchymal transition by interfering with canonical transforming growth factor-beta1-Smad-Snail/Slug axis. Cancer Lett 2014;342:82-91.

-39 Zhang F, Wang H, Wang X, Jiang G, Liu H, Zhang G, Wang H, Fang R, Bu X, Cai S, Du J: TGF-beta induces M2-like macrophage polarization via SNAIL-mediated suppression of a pro-inflammatory phenotype. Oncotarget 2016;7:52294-52306.

40 Lo RS, Massague J: Ubiquitin-dependent degradation of TGF-beta-activated smad2. Nat Cell Biol 1999;1:472-478.

41 Gao S, Alarcon C, Sapkota G, Rahman S, Chen PY, Goerner N, Macias MJ, Erdjument-Bromage H, Tempst P, Massague J: Ubiquitin ligase Nedd4L targets activated Smad2/3 to limit TGF-beta signaling. Mol Cell 2009;36:457-468. 